Document downloaded from:

http://hdl.handle.net/10251/137956

This paper must be cited as:

Sancho-Fornes, G.; Avella-Oliver, M.; Carrascosa Rubio, J.; Morais, S.; Puchades, R.; Maquieira Catala, A. (2019). Enhancing the sensitivity in optical biosensing by striped arrays and frequency-domain analysis. Sensors and Actuators B Chemical. (281):432-438. https://doi.org/10.1016/j.snb.2018.10.130

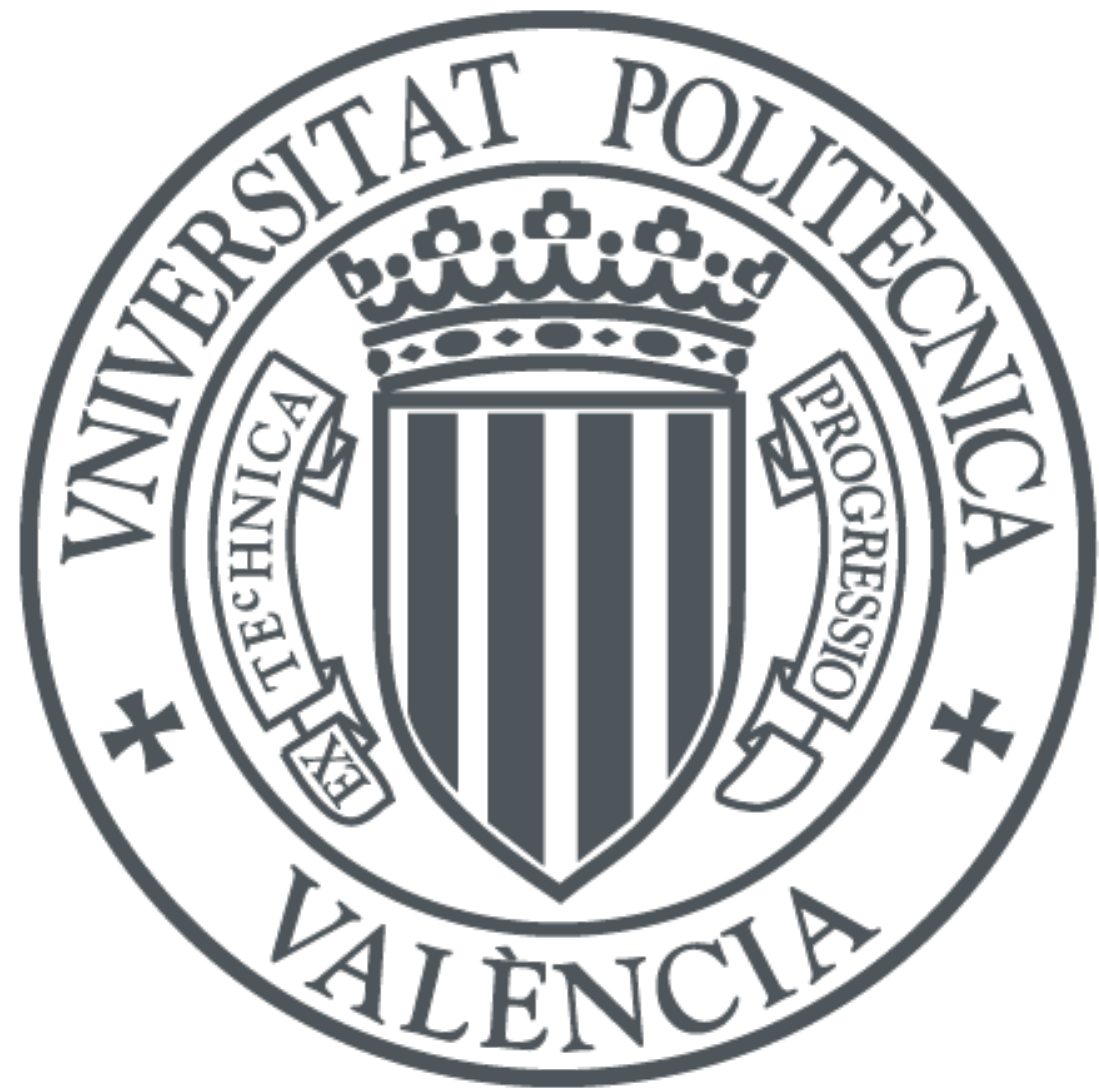

The final publication is available at

https://doi.org/10.1016/j.snb.2018.10.130

Copyright Elsevier

Additional Information 


\title{
Enhancing the Sensitivity in Optical Biosensing by Striped Arrays and Frequency-Domain Analysis
}

\author{
Gabriel Sancho-Fornes ${ }^{a}$, Miquel Avella-Oliver ${ }^{a}$, Javier Carrascosa ${ }^{a}$, Sergi Morais ${ }^{a, b}$, \\ Rosa Puchades ${ }^{a, b}$, and Ángel Maquieira, , $^{a, b, *}$
}

aInstituto Interuniversitario de Investigación de Reconocimiento Molecular y Desarrollo Tecnológico (IDM), Universitat Politècnica de València, Universitat de València, Camino de Vera s/n, 46022 Valencia, Spain.

bDepartamento de Química, Universitat Politècnica de València, Camino de Vera s/n 46022, Valencia, Spain.

${ }^{*}$ Corresponding author.

E-mail address: amaquieira@qim.upv.es

\section{Highlights}

- A simple signal processing based on frequency-domain analysis is introduced.

- Identification and removal of the undesired noise contributions from the analytical signal.

- Approach developed to enhance the sensitivity of optical biosensors.

- Probe of concept determining immunoglobulins and casein through immunoassay.

\section{ABSTRACT}

An approach to enhance the sensitivity of optical biosensors is developed. It is based on patterning strips of biochemical probes and signal processing (frequency-domain analysis, FDA) to the resulting analytical data after a biorecognition assay. This paper introduces FDA, demonstrates for the first time its potential in real bioanalytical systems, describes its critical parameters, and discusses how to optimize them to efficiently exploit this approach for optical biosensing. The results show that FDA enables for the selective identification and removal of the undesired noise contributions 
from the analytical signal arising from a biorecognition event of interest, thus increasing signal-to-noise ratios and, therefore, enhancing the sensitivity of the analysis. In this study, we proof the concept with a model immunassay system for IgGs detection (BSA/anti-BSA) that achieves a sensitivity enhancement of up to 3 orders of magnitude, and then FDA is also demonstrated in a sandwich immunoassay to quantify casein that reaches a limit of detection of $4 \mathrm{ng} / \mathrm{mL}$ (174 pM). Beyond these particular assays, this work also addres to stablish the basis of this approach and to provide keys to stimulate future developments that expand the potential of FDA to other biorecognition assays, transduction systems, and applications.

\section{KEYWORDS}

Optical biosensor, Frequency-domain analysis, Sensitivity enhancement, Immunoassay, Casein.

\section{INTRODUCTION}

Biosensors are booming due to their advantages and performances [1-3], as well as their capability to comply the ASSURED (affordable, sensitive, specific, user-friendly, rapid and robust, equipment free, and deliverable to end users) criteria outlined by the World Health Organization [4]. As in any measurement science, analyzing biorecognition events is also susceptible to signal contributions from external physicochemical sources (noise) which dramatically decreases the sensitivity and reliability of the analysis [5]. Therefore, in addition to optimize the biochemical parameters of the assay [6,7], effective approaches to identify and discriminate the analytical signals of interest are crucial to reach maximal performances in biosensing [8].

Digital filtering (DF) is a widely employed strategy to address this issue in bioanalytical systems [8,9], especially its implementation based on the Fourier transform [10-12]. As schematized in Figure 1a, Fourier transform filters convert the image scanned after the biointeraction into its corresponding frequency counterpart. Then, frequency signals within a selected spectral range (ideally containing the major noise contributions) are attenuated, and the filtered data is finally transformed back to an image. Thought offering good solutions for many analytical applications, these DF strategies keep considerable undesired noise contributions in the resulting data. Moreover, the proper use of DF in chemical analysis typically requires specific studies to identify the 
threshold filtering conditions above which desired physicochemical information is not removed together with the noise [9].

Herein, we present frequency-domain analysis (FDA) as a tool to enhance sensitivity in biorecognition assays by effectively identifying the analytical signals coming from the biointeractions of interest and discriminating them from every noise contribution. As schematized in Figure 1b, FDA relies on patterning (on solid substrates) biorecognition assays as straight and equidistant strips, which generate periodic signals when they are scanned. These periodic signals become unified in a single frequency peak when they are converted into their frequency counterpart using the Fourier transform, whereas undesired contributions are spread along the spectrum. Therefore, by using the intensity of the resulting peak as analytical magnitude, noise can be efficiently discriminated in order to analyze biointeractions in a more sensitive fashion. Schaefer and Chau explored this strategy to discriminate voltage fluctuations in an ink-based optical system [13]. Hereinafter, we introduce for the first time FDA for biochemical analysis, describe and discus the key parameters for its implementation, and experimentally demonstrate the performance improvement provided by this methodology.

Although not directly addressed in this study, it is worth mentioning that the capability of FDA to selectively discriminate the signals coming from the biointeraction of interest, could also be useful to minimize artifacts coming from nonspecific binding in prospective developments [14]. Since the adsorption of nonspecific binding agents tends to present a random (not periodic) distribution along the assay surface, it should display a negligible contribution in the resulting frequency spectrum. Therefore, similarly to the concept behind focal molography and diffraction-based sensing [15-18], this phenomenon could also be potentially exploited by FDA to solve nonspecific binding problems typically observed in the analysis of complex biological matrixes.

On the other hand, FDA is also potentially compatible with any bioanalytical approach based on imaging or scanning biorecognition assays on solid substrates. As a proof of concept, in this paper we implement it in colorimetric biorecognition assays patterned on standard compact disks (as analytical platforms) analyzed by a disk drive tailored to scan the assay response. This is an inexpensive and high-throughput sensing strategy $[19,20]$, herein selected to provide insights into the point-of-care potential of FDA.

As biorecognition assays, in this paper we first address a model immunoassay based on bovine serum albumin to quantify specific lgGs, a well-known system typically used in bioanalysis and herein selected to enhance the representativeness of this study [21]. 
Then, FDA is also applied to a sandwich immunoassay to determine casein. Casein is a family of related phosphoproteins that represents about $80 \%$ of the protein content in milk, whose predominant proteins have a molecular weight of approximately $23 \mathrm{KDa}$. It is estimated that $0.6-2.5 \%$ of children and $1 \%$ of adults have some form of allergy towards dairy products [22], which supports the importance of the quantification and standardization of this target protein. Moreover, both non-processed and digitally filtered (Fourier filter) microarrays are used along the study as reference approaches typically used in the field, in order to compare performances and demonstrate the potential of the new approach herein presented.

\section{MATERIALS AND METHODS}

\subsection{Materials}

Carbonate buffer (50 mM sodium carbonate, $\mathrm{pH}$ 9.6), PBS-T (8 $\mathrm{mM} \mathrm{Na}_{2} \mathrm{HPO}_{4}, 2 \mathrm{mM}$ $\mathrm{KH}_{2} \mathrm{PO}_{4}, 137 \mathrm{mM} \mathrm{NaCl}, 2.7 \mathrm{mM} \mathrm{KCl}, 0.05 \%(v / v)$ Tween-20, $\mathrm{pH}$ 7.4), and washing solutions were filtered through a $0.22 \mu \mathrm{m}$ pore size membranes before use. Bovine serum albumin (BSA), anti-BSA polyclonal antibodies produced in rabbit, gold labeled anti-rabbit polyclonal antibodies produced in goat (GAR-Au), casein, and silver enhancer solutions were supplied by Sigma-Aldrich (Madrid, Spain). Anti-casein and HRP-conjugated anti-casein monoclonal mouse antibodies were kindly provided by Ingenasa S.A. (Madrid, Spain). Tetramethylbenzidine (TMB) substrate was from SDT GmbH (Baesweiler, Germany). Bulk DVD-R disks were purchased from MPO Ibérica (Madrid, Spain). DVD drive (LG HL-DT-ST DVD-RAM GSA-H42N) was from LG Electronics Inc. (Englewood Cliffs, NJ, USA). Silicone-Free adhesive plastic film was purchased from Ultron Systems, Inc (Moorpark, CA, USA). Matt black PVC adhesive sheets were supplied by Aironfix - Eon Paper S.L (Manresa, Spain). Bungard CCD drilling machine was from Bungard (Karo, Germany).

\subsection{Incubation Masks}

To perform the striped patterning of biorecognition assays required for FDA, we developed custom adhesive incubation masks containing striped chambers, to be attached on the assay substrate (Figure $2 \mathrm{a}$ and Figure S1). For that, PVC adhesive sheets and silicone-free adhesive plastic films were cut into $12 \times 12 \mathrm{~cm}$ squares, and they were attached to the non-adhesive part of silicone-free film. The PVC part of this two-layer structure was used to confer firmness to the mask, whereas the silicone-free adhesive plastic was selected since it does not leave residues on the substrate material after its removal. Note that in this case, mask residues on the substrate would 
have same pattern as the striped assay, thus altering the result of the analysis. The masks were patterned with 10 sets of 15 strips each using the drilling machine with a $500 \mu \mathrm{m}$ diameter tungsten carbide drill (feed speed of $800 \mathrm{~mm} \cdot \mathrm{min}^{-1}$ and rotational rate of $30000 \mathrm{rpm})$. The configuration of the masks was designed to generate a set of parallel strips in the resulting scanned image, considering that the employed disk drive scans the platform by following a spiral track at constant linear velocity.

\subsection{BSA Immunoassay}

First, a model immunoassay based on BSA as probes and lgGs as targets was used in this study. For the microarray approach, BSA solutions $(50 \mu \mathrm{g} / \mathrm{mL}$ in carbonate buffer) were arrayed ( $40 \mathrm{~nL} / \mathrm{spot}$ ) on the polycarbonate surface of DVDs using a noncontact printer (AD 1500 Bio-Dot, Inc, Irvine, CA, USA) under controlled temperature and relative humidity $\left(25^{\circ} \mathrm{C}\right.$ and $95 \%$, respectively). On the other hand, to pattern the assays as strips, $1 \mathrm{~mL}$ of BSA ( $50 \mu \mathrm{g} / \mathrm{mL}$ in carbonate buffer) was dispensed onto the disk and distributed using a dummy polycarbonate plate $(12 \mathrm{~cm}$ diameter, $0.6 \mathrm{~mm}$ thick). To attach the proteins on the polycarbonate surface by physical immobilization (passive adsorption) [23], in both cases BSA solutions were incubated for $3 \mathrm{~h}$ at room temperature, and then the disk was washed with PBS-T, rinsed with deionized water, and dried by centrifugation. Afterwards, in the strips patterning protocol, incubation masks were placed onto the disk and a slight pressure was applied in order to attach them.

To perform the selective IgG biorecognition, anti-BSA solutions in PBS-T (40 $\mu \mathrm{L}$ /array in microarrays and $300 \mu \mathrm{L} /$ strip set in FDA) were incubated (20 min, room temperature) in concentrations ranging from $10 \mu \mathrm{g} / \mathrm{mL}$ to $31.6 \mathrm{pg} / \mathrm{mL}$ by serial dilutions of $1 / 10^{0.5}$, including $0 \mathrm{ng} / \mathrm{mL}$. After this incubation, the assay platforms were rinsed with PBS-T, deionized water, and dried by centrifugation. Then, $1 \mathrm{~mL}$ of GAR-Au (1/200 dilution in PBS-T) was dispensed onto the disk, distributed using a dummy polycarbonate plate, incubated for $20 \mathrm{~min}$ at room temperature, and washed and dried again. Finally, $1 \mathrm{~mL}$ of silver enhancer solution was dispensed onto the disk and distributed as before. After $5 \mathrm{~min}$ at room temperature, the disk was washed with deionized water and dried by centrifugation. As a result, insoluble silver precipitates whose surface concentration depends on the magnitude of the biointeraction, are obtained on the assay surface. Real images of exemplary platforms after both microarray and striped assays are shown in Figure S2.

\subsection{Casein Immunoassay}


An immunoassay based on antibodies as probes and casein as target was also studied in this work. To pattern microarrays for this immunoassay, monoclonal anti-casein antibody solutions ( $40 \mu \mathrm{g} / \mathrm{mL}$ in carbonate buffer) were arrayed (40 $\mathrm{nL} / \mathrm{spot})$ on the polycarbonate surface of DVDs using the noncontact printer under controlled temperature and relative humidity $\left(25^{\circ} \mathrm{C}\right.$ and $95 \%$, respectively). To pattern as strips, 1 $\mathrm{mL}$ of monoclonal anti-casein antibody solution ( $40 \mu \mathrm{g} / \mathrm{mL}$ in carbonate buffer) was dispensed onto the disk and distributed using a dummy polycarbonate plate. In both cases, the disks were incubated overnight at $4{ }^{\circ} \mathrm{C}$, and then rinsed with PBS-T and deionized water, and dried by centrifugation.

Afterwards, casein solutions ( $40 \mu \mathrm{L}$ /array for microarrays and $300 \mu \mathrm{L} /$ strip set for FDA) ranging from $100 \mu \mathrm{g} / \mathrm{mL}$ to $100 \mathrm{pg} / \mathrm{mL}$ in PBS-T and blanks, were incubated for $20 \mathrm{~min}$ at room temperature in both cases. Then, the disks were washed, rinsed, and dried as before. Next, $1 \mathrm{~mL}$ of HRP-conjugated anti-casein antibodies (1/20000 dilution in PBS-T) was dispensed onto the disk and distributed using a dummy polycarbonate plate, incubated for $20 \mathrm{~min}$ at room temperature, and washed and dried again. Finally, $1 \mathrm{~mL}$ of TMB substrate solution was dispensed onto the disk and distributed as before. After $8 \mathrm{~min}$ at room temperature, the disk was washed with deionized water and dried by centrifugation. In this case, insoluble dark blue immunoreaction products are generated on the assay surface, whose magnitude is proportional to the concentration of casein.

\subsection{Disk Drive Imaging}

The DVD drive used in this study as analytical scanner was controlled by custom software (BioDisk) running on a personal computer. As described elsewhere [19], the reading process relies on a focused laser beam that scans the disk following an spiral trajectory at linear velocity, which adsorbs and scatters light when it hits the immunoreaction products. This interaction causes variations in the intensity of the reflected laser beam, and these light attenuations correlate with the analyte concentration. Finally, the drive converts these analog signals into binary, and they are sent to the computer and arranged as images.

\subsection{Microarray Data Processing}

As commented before, in this study we assessed FDA by comparing its performance with both raw and digitally filtered (DF) microarrays as reference methods. Raw microarray images were directly generated by the disk drive imaging system, as described above. On the other hand, to obtain the DF microarrays, raw images were processed with a custom Matlab function (MathWorks, Inc., Natick, MA, USA) to apply 
the Fast Fourier transform and process their frequency spectra, as schematized in Figure 1a. Different threshold filtering values were assessed, which removed nearly half of the noise and kept close to constant assay signals (Table S1), thus improving the sensitivity of the system. From these results, $100 \mathrm{KHz}$ was selected as a threshold filtering value to attenuate the high-frequency noise in DF microarrays.

GenePix software (Axon Inst, Union City, CA, USA) was used to quantify signals of both raw and DF microarrays. Analytical signals of each array were calculated by averaging the intensity of all the replicated microarray spots and subtracting their background. Noise values were experimentally obtained as the standard deviation of 10 blank replicates ( $0 \mathrm{ng} / \mathrm{mL}$ of target), and signal-to-noise ratios (SNR) were calculated by dividing the signal of each assay between its noise.

\subsection{FDA Data Processing}

Scanning the biorecognition assays patterned as strips on the assay surface leads to images constituted by sets of parallel lines, as observed in Figure $1 \mathrm{~b}(\mathrm{i})$ and Figure 2. To process these images, they were first cropped into $15 \mathrm{~mm}$ width and $4 \mathrm{~mm}$ height rectangles with Photoshop CS4 (Adobe Systems Inc., San Jose, CA, USA). Next, a custom Matlab algorithm was used to apply the Fast Fourier transform to the scanned images, in order to obtain the frequency spectrum of every data row (Figure $1 \mathrm{~b}(\mathrm{ii})$ ) and to average all of them in a single one, from which using the height of the resulting peak as analytical signal (Figure $1 \mathrm{~b}(\mathrm{iii})$ ). In the FDA approach, experimental noise values were calculated as the standard deviation of the peak intensity of ten blank replicates.

The employed Matlab algorithm also transforms the magnitude in the horizontal axis of the resulting frequency spectra, from frequency to period (distance). This transformation enables to visualize the resulting FDA peak centered in the period value in which the striped biorecognition assays are patterned. In this system, the correlation of frequency and period is described by the equation below:

$$
\text { Period }=\frac{\text { sampling rate } \cdot \text { resolution }}{\text { frequency }}
$$

where sampling rate is the number of measurements per time unit took by the scanning system, and resolution is the length of the assay substrate scanned by each one of these measurements. The sampling rate and the resolution of the disk drive scanner employed in this study are $2000 \mathrm{KHz}$ and $6.98 \mu \mathrm{m} /$ pixel, respectively. As a result, with the incubation mask herein developed, FDA signals were mainly concentrated at 13918 $\mathrm{Hz}$ in frequency, which corresponds to $1 \mathrm{~mm}$ in period. 


\section{RESULTS AND DISCUSSION}

\subsection{FDA Parameters}

In this section we describe the main parameters that affect FDA and discuss how to deal with them in order to fully exploit the analytical performance of this approach. First, the width of the assay strips and the separation between them were investigated. These two parameters (together with the features of the analytical scanner) determine the resulting profile of the patterned assays in the scanned image, which strongly affects the FDA response. As shown Figure S3, sinusoid profiles concentrate the signal in a single (fundamental) peak, whereas other profiles (such as squared and triangular) distribute the signal also in additional harmonic peaks.

To achieve a sinusoid profile, the width of the strips and the separation between them have to be the same, namely, the duty cycle must be $50 \%$. On the other hand, as inferred from simulations considering the optical resolution of the scanning system employed in this study (Figure S4 and Eq. S1), the desired sinusoid profile can be obtained by patterning $500 \mu \mathrm{m}$ width assays strips within $1 \mathrm{~mm}$ periods.

Different methods were investigated to experimentally create these striped patterns of proteins on the polycarbonate surface of the assay substrates, such as non-contact printing and soft lithography $[24,25]$. Among them, the best results were obtained by creating masks with striped holes, and then attaching them on the assay surface in order to incubate biochemicals in a patterned fashion (Figure 2a). These masks were initially made of PDMS and PVC adhesive sheet, but they left residues on the assay surface after their removal, and these residues became patterned in the gaps between the assay strips. Since gaps and strips have the same period, the signal contributions coming from both parts were unified in the frequency peak, which increases the resulting noise and reduces the performance of FDA. To avoid this handicap, siliconefree adhesive plastic film was selected to create the incubation masks (Figure S1). This material does not leave residues on the assay substrate after its removal, and it enables a clean patterning process that results in well-defined assay strips with the desired sinusoid profile in the scanned image (Figure $2 b-c$ ).

The number of strips is another important parameter in the FDA response. As theoretically (Figure S5) and experimentally (Figure 3a) observed, frequency peaks become narrower when the number of strips increase. Moreover, the resolution of the frequency spectrum improves together with this number (Figure 3a). The stepwise shape of this curve is due to the Fast Fourier transform used to obtain the frequency spectra, which allows to calculate the discrete Fourier transform quickly and with 
minimal computational requirements. This method rounds up the number of processed data to the next power of 2, and each step in Figure 3a corresponds to one of those values in the number of pixels in each row of the scanned image. The number of strips becomes especially important in the design of prospective multiplexed FDA systems based on assay strips patterned according to different periods (to sense different targets), thus generating distinct frequency peaks for each analyte in a single FDA assay.

Therefore, since higher spectral resolutions and narrower peaks favor FDA analysis (especially for multiplexed FDA), the potential sensitivity to be achieved scales up together with the number of strips, and consequently with the area employed by the assay. On the other hand, a greater number of strips require higher volumes of bioreagents and samples. In high-resolution bioanalytical scanning systems, this issue can be easily addressed by decreasing the size of the strips. A set of 15 strips was chosen in this study, since it is a smaller number that involves great resolution and narrow peaks.

The length of the strips was also investigated. The importance of this parameter lies in the fact that the frequency peaks also comprise small contributions of random noise. Since FDA averages frequency spectra from every raw of data (Figure 1b), the longer the strips are the larger number of scanned rows, and consequently, the noise (the standard deviation) decreases with the square root of the length, according to the Bienaymé formula [26]. The results in Figure $3 b$ show that the noise remains rather constant after $4 \mathrm{~mm}$ of strip length, so this value was selected for the subsequent experiments.

As presented in Figure 4, the expected FDA result was successfully obtained with the optimized assay patterning conditions. Despite the sinusoidal shape of the scanned assay strips, very weak harmonic peaks are also observed in the spectra, which may be generated by minor profile irregularities in the resulting experimental strips. However, the intensity of these harmonics is negligible compared to the one of the fundamental peak. It must be noted that we used distance magnitudes instead of frequencies for the horizontal axis of the resulting FDA spectra. For optical setups that scan the assay substrate at constant velocity, both variables have an inverse and linear correlation (Eq. 1). However, using distances provides intuitive and practical FDA readouts, in which the fundamental peak appears centered in the distance value that corresponds to the period of the patterned strips (1 $\mathrm{mm}$ in this case), as observed in Figure 4. 
We also investigated the effect, on the FDA performance, of random and systematic errors in the assays patterning. For that, we modelled different modifications of the strips and assessed their repercussion in the resulting frequency peak. As an example of a random error, we considered the absence of strips randomly distributed along the strips set. It represents an extreme case for a random patterning error that diminish the averaged intensity of particular strips within a set. For instance, in the present work, this error could come from imperfections when filling the chambers with the probe solution. As shown Figure S6, these random errors affect the resulting FDA response, and the intensity of the resulting frequency peak decreases linearly with the number of missing strips. Also, it is worth to note that the position of the removed strips presents a negligible repercussion in the resulting peak intensity (RSD $<0.1 \%$ in all cases).

On the other hand, as a systematic patterning error, we considered a systematic deviation of the strip widths along the whole set of strips. For example, this error could come from experimental deviations in the method employed to create the striped pattern. In this particular development, it could be generated by inaccuracies in the diameter of the drill used to pierce the incubation masks. As shown in Figure S7, this systematic modification of the strips width modifies the intensity of the frequency peak according to a Gaussian trend. Although this intensity becomes suppressed in both extremes of the curve, it is worth to highlight that it only decreases less than $2 \%$ when the width varies up to a $10 \%( \pm 50 \mu \mathrm{m}$ in this work) around the central width value selected to obtain the desired sinusoid profile.

\subsection{Model Immunoassay System}

In order to experimentally assess the performance of FDA in real assays, this strategy was compared with raw (non-filtered) and digitally filtered (DF) microarrays. Raw microarrays were obtained directly from the imaging system, their DF counterparts were processed with a low pass Fourier filter using optimized cut-off values to remove high-frequency noise (Table S1), and FDA was performed as described in the previous section.

For this comparison, we used a model immunoassay system based on immobilizing BSA on the assay surface and quantifying selective lgGs in solution. A wide range of lgG concentrations were analyzed by all the approaches in the same conditions.

Sensitivity was evaluated by means of SNRs, and limits of detection (LOD) and quantification (LOQ) were considered as the experimental IgG concentration associated with $\mathrm{SNR}=3$ and $\mathrm{SNR}=10$, respectively. 
As observed in Figure 5, well-correlated dose-response curves were obtained in all the cases. More interestingly, raw microarrays display the lowest SNR values, DF microarrays slightly increase SNRs, and FDA approach exhibits a dramatic improvement that has an impact on the LOD and LOQ. Table 1 summarizes the analytical parameters obtained from these experimental dose-response curves. It can be observed that DF leads only to a two-fold noise reduction compared to raw microarray. However, FDA achieves a close-to-zero noise and reaches a sensitivity enhancement of about 2-3 orders of magnitude for this model immunoassay system, which enables the detection of $30 \mathrm{pg} / \mathrm{mL}(200 \mathrm{fM})$ of $\mathrm{lgG}$.

\subsection{Casein Immunoassay}

After proving the concept of FDA with a model system, this section aims to demonstrate the potential of this strategy in other immunoassays, and to provide insights into real applications to analyze food allergens. For that, we applied FDA to a sandwich immunoassay to quantify casein. Instead of using gold-labelled secondary antibodies together with silver signal enhancement as in the previous section, the signal development was here based on HRP-labelled primary antibodies followed by TMB precipitation.

In this study, the performance of FDA for this casein immunoassay was assessed and compared to raw microarrays. DF microarrays were omitted in this assessment, since this strategy displayed a poor improvement in the previous section. As before, sensitivity was evaluated by means of SNRs, and LOD and LOQ were considered as the target concentration corresponding to $S N R=3$ and $S N R=10$, respectively.

As observed in Figure 6, both dose-response curves display the expected sigmoidal response, and the characteristics (binding affinities and signal developing strategy) of this casein immunoassay lead to lower signals than the previous BSA immunoassay. The graph shows that, also in lower signal conditions, FDA generates significant SNR enhancements in the dose-response curve. As summarized in Table 2, FDA reduces the noise and improves LOD about two orders of magnitude compared to the raw microarray assay format, reaching a sensitivity of $4 \mathrm{ng} / \mathrm{mL}$ (174 pM) of casein.

\section{CONCLUSION}

FDA is a practical approach to reduce noise and improve sensitivity in optical biosensing. As experimentally demonstrated in this study in two different systems, the sensitivity of biorecognition assays patterned as strips on solid substrates boosts up to 
2-3 orders of magnitude by just applying a simple signal processing algorithm, compared to reference microarray systems. FDA must potentially provide higher sensitivity enhancements in bioanalytical systems comprising greater contributions of unwanted signals, such as in simple and low-cost biosensing setups. Therefore, this approach entails important strengths to reduce the resources required to develop biosensors without sacrificing their analytical performance, which suggests simpler and cheaper systems for point-of-care analysis. Besides, the capability of FDA to reject unwanted signals introduces appealing perspectives to develop new bioanalytical systems that provide solutions to the problems associated with nonspecific binding in the analysis of complex matrixes. Also, the multiplexing potential of FDA could be enhanced by patterning (in different periods) multiple biorecognition assays on the same substrate area, and then discriminating their FDA peaks in the frequency spectrum. Moreover, our results point towards prospective FDA advances to develop highly sensitive assays exploiting different optical events (fluorescence, light interference, SPR, etc.) applied to determine a wide range of targets (proteins, DNA, cells, small molecules, etc.).

\section{ASSOCIATED CONTENT}

Supplementary Material

\section{NOTES}

The authors declare no competing financial interest.

\section{ACKNOWLEDGMENT}

This research was supported by FEDER and the Spanish Ministry of Economy and Competitiveness (CTQ2016-75749-R). 


\section{REFERENCES}

[1] R. Huang, N. He, Z. Li, Recent progresses in DNA nanostructure-based biosensors for detection of tumor markers, Biosens. Bioelectron. 109 (2018) 2734. doi:10.1016/j.bios.2018.02.053.

[2] A. Tereshchenko, M. Bechelany, R. Viter, V. Khranovskyy, V. Smyntyna, N. Starodub, R. Yakimova, Optical biosensors based on ZnO nanostructures: Advantages and perspectives. A review, Sens. Actuators B. 229 (2016) 664677. doi:10.1016/j.snb.2016.01.099.

[3] C.I.L. Justino, A.C. Duarte, T.A.P. Rocha-Santos, Critical overview on the application of sensors and biosensors for clinical analysis, Trends Anal. Chem. 85 (2016) 36-60. doi:10.1016/j.trac.2016.04.004.

[4] H. Kettler, K. White, S. Hawkes, Mapping the landscape of diagnostics for sexually transmitted infections: Key findings and recommandations, Unicef/Undp/World Bank/Who. (2004) 1-44.

[5] V.A. Galievsky, A.S. Stasheuski, S.N. Krylov, Improvement of LOD in Fluorescence Detection with Spectrally Nonuniform Background by Optimization of Emission Filtering, Anal. Chem. 89 (2017) 11122-11128. doi:10.1021/acs.analchem.7b03400.

[6] M. Avella-Oliver, D. Gimenez-Romero, S. Morais, M.Á. González-Martínez, P.R. Bueno, R. Puchades, Á. Maquieira, INSEL: an in silico method for optimizing and exploring biorecognition assays, Chem. Commun. 49 (2013) 10868-10870. doi:10.1039/c3cc44018d.

[7] W.-J. Kim, H.Y. Cho, B.K. Kim, C. Huh, K.H. Chung, C.-G. Ahn, Y.J. Kim, A. Kim, Highly sensitive detection of cardiac troponin I in human serum using gold nanoparticle-based enhanced sandwich immunoassay, Sens. Actuators B. 221 (2015) 537-543. doi:10.1016/j.snb.2015.06.128.

[8] P.D. Wentzell, C.D. Brown, Signal Processing in Analytical Chemistry, in: Encylclopedia Anal. Chem., John Wiley \& Sons, Ltd, Chichester, UK, 2006: pp. 9764-9800. doi:10.1002/9780470027318.a5207.

[9] M. Avella-Oliver, S. Morais, J. Carrascosa, R. Puchades, Á. Maquieira, Total analysis systems with thermochromic etching discs technology, Anal. Chem. 86 (2014) 12037-12046. doi:10.1021/ac502640j.

[10] V.J. Barclay, R.F. Bonner, I.P. Hamilton, Application of wavelet transforms to 
experimental spectra: Smoothing, denoising, and data set compression, Anal. Chem. 69 (1997) 78-90. doi:10.1021/ac960638m.

[11] Jean-Luc Starck, E.J.J. Candes, D.L.L. Donoho, J.L. Starck, E.J.J. Candes, D.L.L. Donoho, The curvelet transform for image denoising, IEEE Trans Image Process. 11 (2002) 670-684. doi:10.1109/TIP.2002.1014998.

[12] Y. Tan, A. Chu, M. Lu, B.T. Cunningham, Distributed Feedback Laser Biosensor Noise Reduction, IEEE Sens. J. 13 (2013) 1972-1978. doi:10.1109/JSEN.2013.2244591.

[13] S. Schaefer, K.J. Chau, Improving the signal visibility of optical-disk-drive sensors by analyte patterning and frequency-domain analysis, Meas. Sci. Technol. 22 (2011) 045302. doi:10.1088/0957-0233/22/4/045302.

[14] J.-F. Masson, T.M. Battaglia, J. Cramer, S. Beaudoin, M. Sierks, K.S. Booksh, Reduction of nonspecific protein binding on surface plasmon resonance biosensors, Anal. Bioanal. Chem. 386 (2006) 1951-1959. doi:10.1007/s00216006-0834-2.

[15] C. Fattinger, Focal molography: Coherent microscopic detection of biomolecular interaction, Phys. Rev. X. 4 (2014) 31024. doi:10.1103/PhysRevX.4.031024.

[16] V. Gatterdam, A. Frutiger, K.-P. Stengele, D. Heindl, T. Lübbers, J. Vörös, C. Fattinger, Focal molography is a new method for the in situ analysis of molecular interactions in biological samples, Nat. Nanotechnol. 12 (2017) 1089-1095. doi:10.1038/nnano.2017.168.

[17] M. Avella-Oliver, J. Carrascosa, R. Puchades, Á. Maquieira, Diffractive protein gratings as optically active transducers for high-throughput label-free immunosensing, Anal. Chem. 89 (2017) 9002-9008. doi:10.1021/acs.analchem.7b01649.

[18] M. Avella-Oliver, V. Ferrando, J.A. Monsoriu, R. Puchades, A. Maquieira, A label-free diffraction-based sensing displacement immunosensor to quantify low molecular weight organic compounds, Anal. Chim. Acta. 1033 (2018) 173-179. doi:10.1016/J.ACA.2018.05.060.

[19] S. Morais, R. Puchades, Á. Maquieira, Disc-based microarrays: principles and analytical applications, Anal. Bioanal. Chem. 408 (2016) 4523-4534. doi:10.1007/s00216-016-9423-1.

[20] M. Avella-Oliver, R. Puchades, S. Wachsmann-Hogiu, A. Maquieira, Label-free 
SERS analysis of proteins and exosomes with large-scale substrates from recordable compact disks, Sens. Actuators B. 252 (2017) 657-662. doi:10.1016/j.snb.2017.06.058.

[21] A. Kooser, K. Manygoats, M.P. Eastman, T.L. Porter, Investigation of the antigen antibody reaction between anti-bovine serum albumin (a-BSA) and bovine serum albumin (BSA) using piezoresistive microcantilever based sensors, Biosens. Bioelectron. 19 (2003) 503-508. doi:10.1016/S0956-5663(03)00221-5.

[22] J. Ashley, M. Piekarska, C. Segers, L. Trinh, T. Rodgers, R. Willey, I.E. Tothill, An SPR based sensor for allergens detection, Biosens. Bioelectron. 88 (2017) 109-113. doi:10.1016/j.bios.2016.07.101.

[23] F. Rusmini, Z. Zhong, J. Feijen, Protein Immobilization Strategies for Protein Biochips, Biomacromolecules. 8 (2007) 1775-1789. doi:10.1021/bm061197b.

[24] C. Ru, J. Luo, S. Xie, Y. Sun, A review of non-contact micro- and nano-printing technologies, J. Micromechanics Microengineering. 24 (2014) 053001. doi:10.1088/0960-1317/24/5/053001.

[25] J. Voskuhl, J. Brinkmann, P. Jonkheijm, Advances in contact printing technologies of carbohydrate, peptide and protein arrays, Curr. Opin. Chem. Biol. 18 (2014) 1-7. doi:10.1016/j.cbpa.2013.10.022.

[26] D.P. Griesheimer, V.S. Sandhu, Analysis of inconsistent source sampling in monte carlo weight-window variance reduction methods, Nucl. Eng. Technol. 49 (2017) 1172-1180. doi:10.1016/J.NET.2017.07.017.

[27] C.T. Goudar, K. Joeris, K.B. Konstantinov, J.M. Piret, Logistic equations effectively model mammalian cell batch and fed-batch kinetics by logically constraining the fit, Biotechnol. Prog. 21 (2005) 1109-1118. doi:10.1021/bp050018j.

[28] J. Schiettecatte, E. Anckaert, J. Smitz, Interferences in immunoassays, in: Adv. Immunoass. Technol., InTech, 2012: pp. 45-62. doi:10.5772/35797. 


\section{Figure captions}

(a)
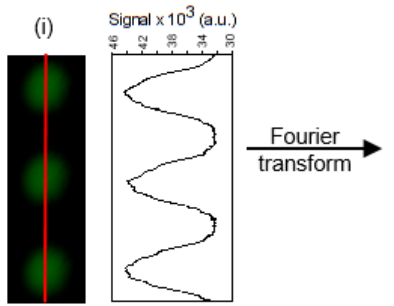

(ii)
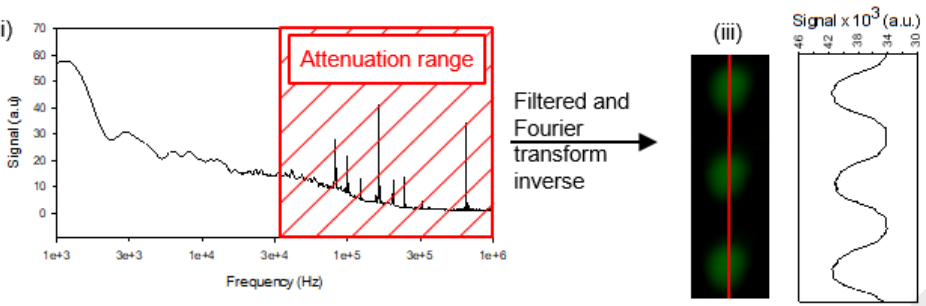

(b)
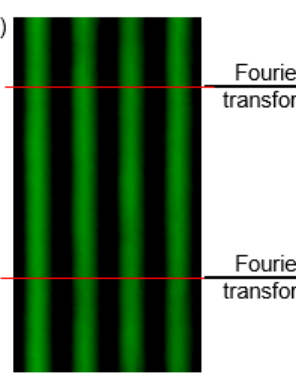

(ii)
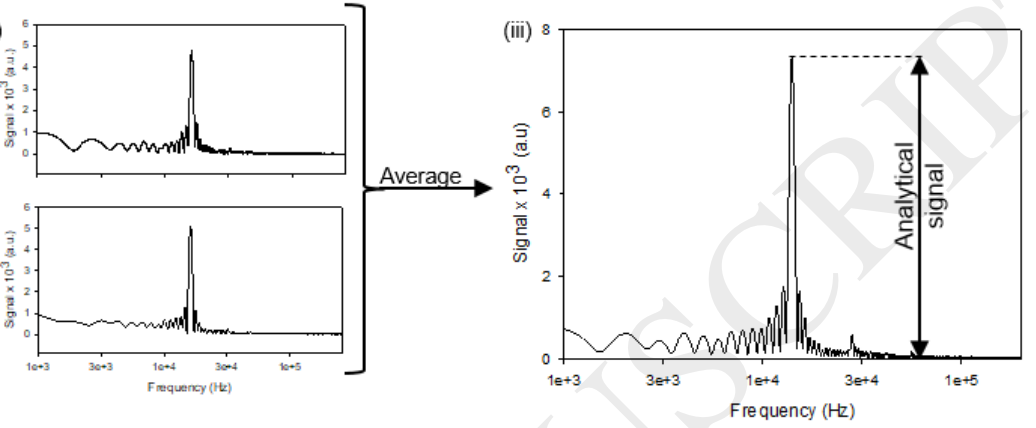

Figure 1. Scheme of the signal processing approaches studied in this work. (a) Fourier digital filtering: (i) scanned raw microarray image and its cross section, (ii) the corresponding frequency spectrum obtained by Fourier transform, which is filtered to attenuate high-frequency contributions, and (iii) the resulting filtered microarray image and its cross section. (b) FDA approach: (i) scanned image of the patterned assay strips, (ii) two exemplary frequency spectra of single data rows, and (iii) the resulting frequency spectrum generated by averaging the one of every data row. 


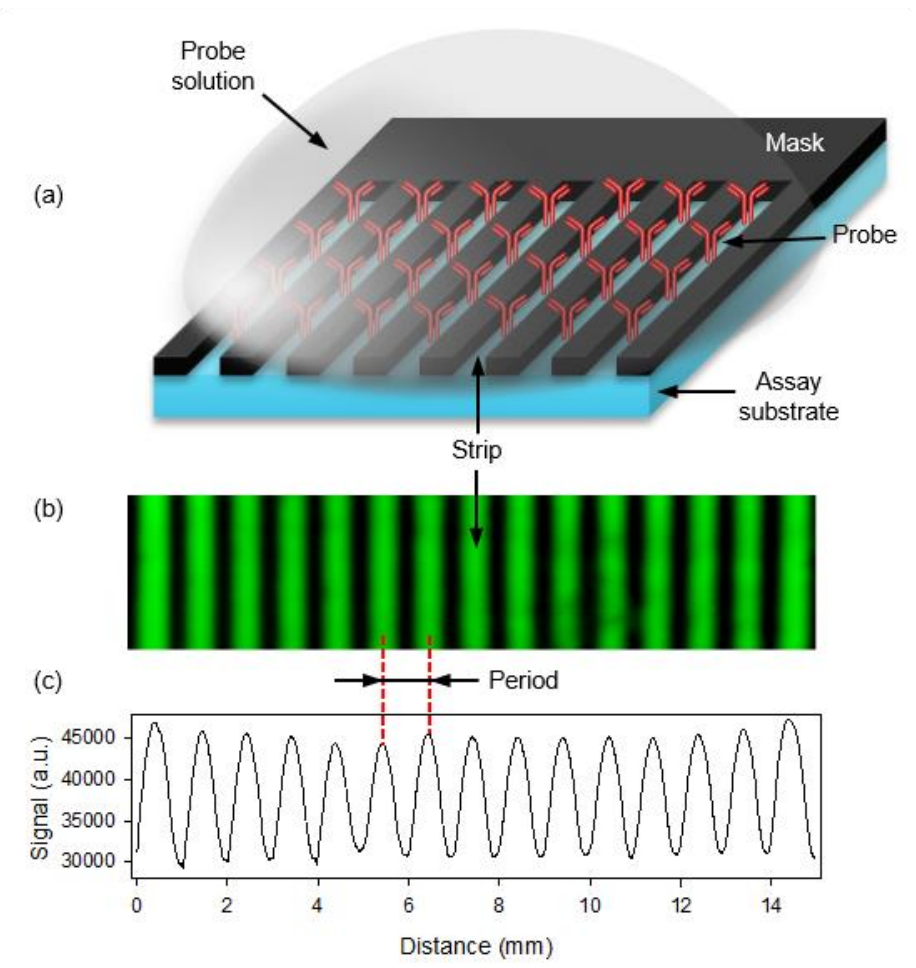

Figure 2. Striped patterning of biorecognition assays. (a) Schematic representation of the patterning of biological probes using the adhesive incubation mask with striped chambers herein developed. (b) Scanned image experimentally obtained from a patterned BSA immunoassay and (c) its corresponding sinusoidal-shaped averaged profile.
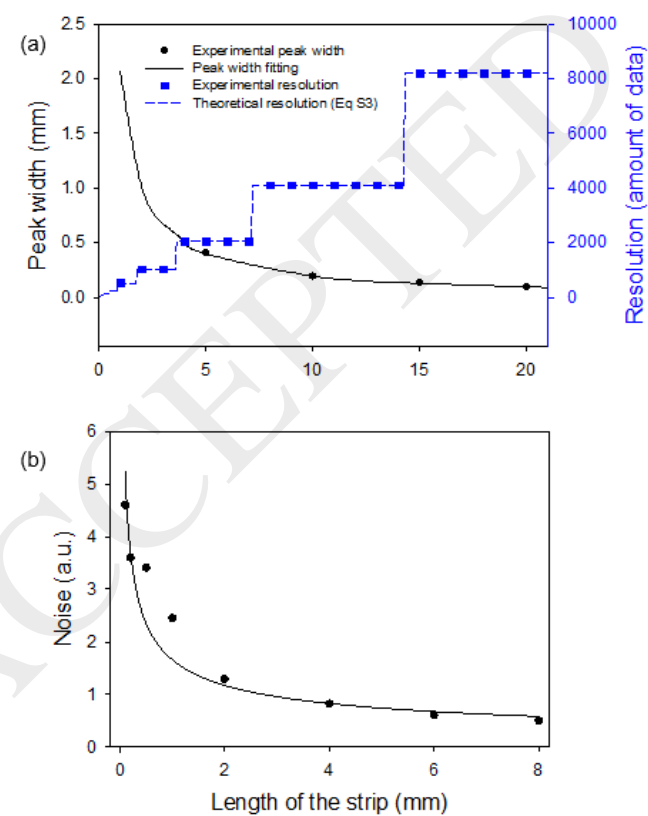

Figure 3. Number of strips and their length. (a) Experimental curve of number of strips versus both peak width and resolution in the frequency spectrum. Data fitted to Equation S2 $\left(R^{2}=0.999\right)$ for peak width and compared to Equation S3 for theoretical resolution. (b) 
Experimental results of the effect of the strips length on the resulting noise in the FDA analysis. Data fitted to Bienaymé formula $\left(R^{2}=0.872\right)$.

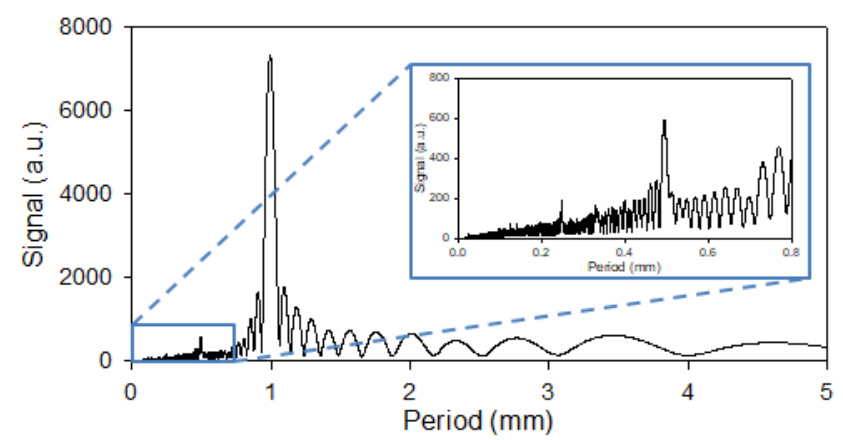

Figure 4. Exemplary experimental frequency spectrum for a patterned BSA immunoassay, representing period values in the horizontal axis. See Figure $2 b$ and $2 c$ for the scanned image counterpart of this spectrum. Inset graph zooms in in the short period range, to show the harmonic peaks.

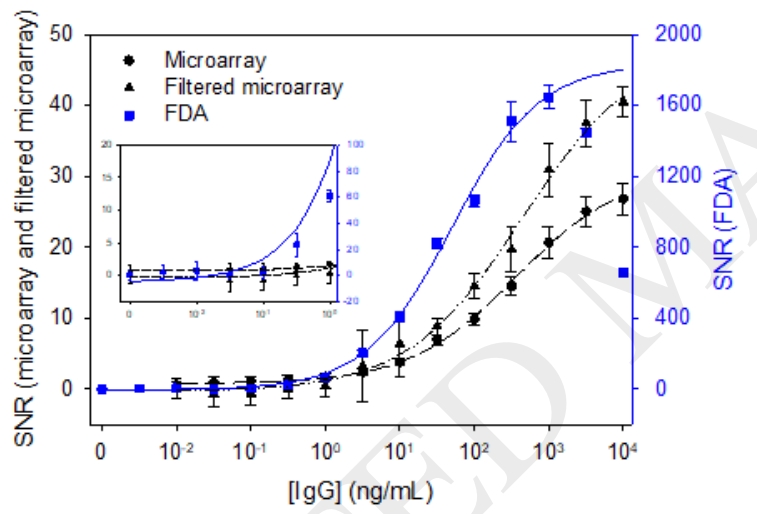

Figure 5. Dose-response curves for the FDA assessment with a model immunoassay system. Note the two different SNR scales in this graph. Experimental data fitted to four-parameter logistic equation $\left(R^{2}\right.$ raw $=0.998, R^{2} D F=0.995$, and $\left.R^{2} F D A=0.997\right)$ [27]. The two points within the hook effect area observed at high IgG concentrations in the striped dose-response curve were omitted in the fitting process [28]. Inset plot focuses on the LOD and LOQ zones. In this experiment, 15 replicated spots were analyzed for raw and DF microarray, and sets of 15 strips were used for FDA. 


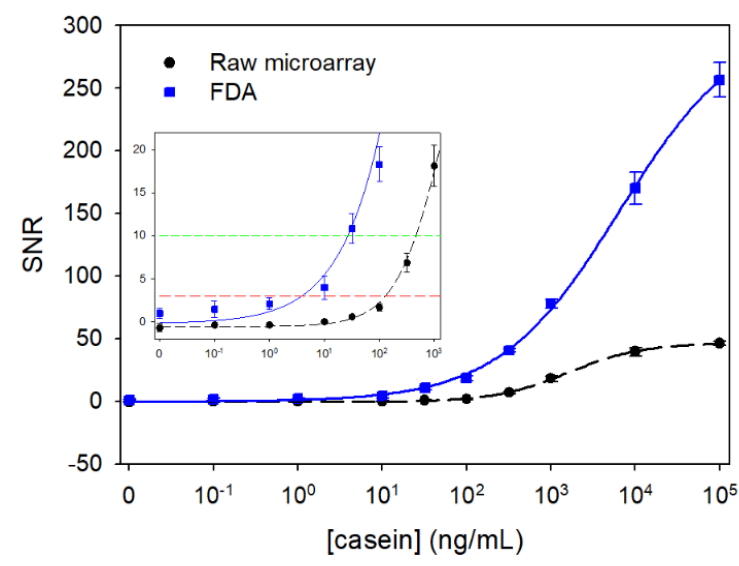

Figure 6. Dose-response curves for the FDA assessment with a sandwich immunoassay for casein analysis. Experimental data fitted to four-parameter logistic equation $\left(R^{2}=0.999\right.$ in both fits). Inset plot zooms in in the LOD and LOQ concentration ranges, where the green and red dashed lines mark the SNR thresholds for LOQ and LOD, respectively. In this experiment, averaged values and standard deviations were calculated from 3 replicated microarray dots and three sets of 15 FDA strips. 
Table

Table 1. Analytical parameters for the FDA assessment with the model immunoassay system.

\begin{tabular}{|l|l|l|l|l|}
\hline & noise (a.u.) & max. SNR & LOQ $(\mathrm{ng} / \mathrm{mL})$ & LOD $(\mathrm{ng} / \mathrm{mL})$ \\
\hline Raw microarray & 218 & 30 & 95 & 6 \\
\hline DF microarray & 114 & 49 & 42 & 4 \\
\hline FDA & 4 & 1840 & 0.08 & 0.03 \\
\hline
\end{tabular}

aSNR value at the steady state of the logistic fitting.

Table 2. Analytical parameters for the FDA assessment with the sandwich immunoassay for casein analysis.

\begin{tabular}{|l|l|l|l|l|l|}
\hline & noise (a.u.) & max. SNR & \multicolumn{2}{l|}{ LOQ $(\mathrm{ng} / \mathrm{mL})$} & \multicolumn{2}{l|}{ LOD $(\mathrm{ng} / \mathrm{mL})$} \\
\hline Raw microarray & 177 & 47 & 462 & 129 \\
\hline FDA & 6 & 303 & 27 & & \\
\hline
\end{tabular}

